\title{
Author's reply to "Comments on "Treatment of anal fistula with FiLaC: results of a 10-year experience with 175 patients"'' A. Anand, A. K. Jha, M. Kumar
}

\author{
P. Giamundo ${ }^{1}$ - M. De Angelis ${ }^{1}$
}

Received: 5 February 2022 / Accepted: 28 February 2022 / Published online: 8 March 2022

(c) Springer Nature Switzerland AG 2022

\section{Dear Sir,}

We want to thank Dr. Anand and colleagues [1] for their interest in our article on the treatment of anal fistulas with laser [2].

We would like to reply to the points they made:

1. Talking about surgical treatments of anal fistula, we still believe one of the problems influencing objective evaluation of different surgical techniques is the lack of homogeneity of the type of fistulas being treated. We agree that our study too was carried out on different types of fistulas. In fact our comment was a general observation and does not exclude our study.

2. It was clearly specified in "Materials and Methods" that preoperative evaluation included endorectal ultrasound (ERUS) or magnetic resonance imaging (MRI) in all patients in our study. (MRI was indicated in patients with multiple orifices or those with repeatedly recurring fistulas, ERUS in the others). One hundred and forty-two patients out of 175 had seton or loop in place after the first stage operation. All of them underwent a second MRI (or ERUS) before the laser treatment [fistula laser closure (FilaC)] in order to exclude persisting abscesses or secondary, residual fistula tracts.

3. Again, an MRI or ERUS was performed preoperatively in all 175 patients included in the study (not only in 142 out of 175).

4. 'Other surgical procedures' prior to laser treatment included: over-the-scope clip (OTSC), brushing/ curettage or a mixture of different procedures such as flaps + plugs or flaps + stem cells.

\footnotetext{
P. Giamundo pgiamundo@gmail.com

1 Policlinico di Monza, Monza, Italy
}

5. In the results section, Anand et al. are right to point out that we mentioned that the internal orifice was sutured in 9 out of 58 patients instead of 7 out of 58 , although in Table 4 the correct figures are reported.

6. "Advancement flap" was performed in 5 patients in whom FilaC had failed. This procedure was not in the details described in the Methods section as the procedure was not different from the endorectal advancement flap described in most papers in the literature.

\section{Declarations}

Conflict of interest Paolo Giamundo, first and corresponding author, declares that he has no conflict of interest.

Ethical approval This article does not contain any studies with human participants or animals performed by any of the authors.

Informed consent For this type of study formal consent is not required.

\section{References}

1. Anand A, Jha AK, Kumar M (2021) Comments on "Treatment of anal fistula with FiLaC®: results of a 10-year experience with 175 patients." Tech Coloproctol. https://doi.org/10.1007/ s10151-021-02553-1

2. Giamundo P, De Angelis M (2021) Treatment of anal fistula with FiLaC $®$ : results of a 10-year experience with 175 patients. Tech Coloproctol 25(8):941-948. https://doi.org/10.1007/s10151-02102461-4 (Epub 2021 May 19)

Publisher's Note Springer Nature remains neutral with regard to jurisdictional claims in published maps and institutional affiliations. 